\title{
Research on the Integration Mechanism of Women's Volleyball Spirit and Physical Education Curriculum
}

\author{
Yongle Qi \\ College of Physical Education and Health Science, Zhejiang Normal University, Zhejiang, China
}

\begin{abstract}
From the perspective of core literacy, the core elements of sports are relatively complex. In order to better reflect the practical significance of sports core literacy in physical education curriculum and give full play to the role of moral education and talent cultivation of physical education curriculum, this paper uses the methods of literature and logical analysis, under the framework of sports core literacy model, takes the women's volleyball spirit as the value guidance, combined with the nature of physical education curriculum, from the perspective of disciplinary physical education curriculum and active physical education curriculum, to explore the mechanism of the integration of women's volleyball spirit and physical education curriculum.
\end{abstract}

Keywords: Women's volleyball spirit, Core literacy.

\section{Introduction}

Core literacy is the inheritance and Transcendence of the "three-dimensional" goal after the new curriculum standard puts forward the "three-dimensional" goal. The "core quality" defined in China refers to "the personality quality and key ability gradually formed by students in the process of receiving the education of the corresponding school period to adapt to personal lifelong development and social development"[1]. The proposal of core literacy provides relevant theories for the development of school curriculum. The future trend and development of physical education curriculum should also meet the connotation requirements of core literacy. Only in this way, physical education curriculum can keep pace with the trend of the times and adapt to school reform. Based on this, Ms. Zhao studied the construction of sports core literacy model close to the characteristics of sports discipline. The sports core literacy model is also called "fish bone" model because its arrangement is similar to fish bone. Taking the socialist core values as the core, this model constructs a sports core literacy model with Chinese characteristics from 11 aspects of three categories: man and tools, man and self-development, man and society.

\section{Theoretical Framework}

\subsection{The Core Literacy and the Concept of Sports Core Literacy}

Countries all over the world have conducted some research based on core literacy and achieved important results. In 1997, the organization for economic cooperation and development constructed a parallel and interactive core literacy model from three dimensions: man and tool, man and self, man and society; Singapore has built a concentric core literacy model from the three dimensions of core, middle core and outer core; The concentric circle model of " 21 st century capability" in Japan constructs the core literacy model from three aspects: foundation, thinking and practice; The construction of these models has common requirements in students' personal value performance, social participation and responsibility, learning ability and so on, but they have characteristic performance in the composition of internal elements due to national and regional differences. If the core quality is a "blueprint" outlined as the new image expected in the new era, then each discipline is the "component" to support the realization of this blue picture. They each have their inherent essential characteristics, basic concepts and skills, as well as the cognitive mode, thinking mode and representation mode embodied by their respective disciplines. Based on this, Zhao Fengxia, in the research on the construction of sports core literacy model, constructed the sports core literacy model and put forward the concept of sports core literacy: students acquire and construct a series of sports knowledge through organized physical education teaching and students' independent, cooperative and creative learning and exercise within the scope of the school The dynamic process of sports technology and sports skills, and in this process, gradually form the consciousness and ability of healthy development of lifelong sports, as well as the sports spirit and personality characteristics of brave struggle, unity and cooperation, optimism and self-confidence to better adapt to social development[2].

\subsection{Combination Point of People and Tools in Physical Education Curriculum}

Volleyball was invented by William Morgan in 1895 and has a history of 125 years. It has been 115 years since it was introduced to China in 1905. After the tortuous development of the initial 16-player game system to 12-player game system, and the 9-player game system to the current 6-player game system, volleyball plays a huge role in influence, competitive performance, and application in physical education courses. As a tool, volleyball is the link between students and the classroom; it is also the material for compiling the content of physical education courses and completing the course goals. The women's volleyball team, as the representative of sports in the new era in China, has condensed the spirit of hard work and unity, and these spirits have had a huge impact on today's society. The spirit of women's volleyball is integrated into the physical education curriculum and becomes the link between the sports tools and people in the physical education curriculum. It not only provides a concrete basis for the 
practice of the physical education curriculum, but also makes the physical education curriculum goals have more specific connotations.

\subsubsection{Teachers and students build a sports classroom}

The spirit of the women's volleyball team is integrated into the physical education curriculum, enabling teachers and students to build a physical education classroom together. In the physical education curriculum, whether it is a subject physical education curriculum or an active physical education curriculum, the existing venue equipment is a major factor restricting its development. Venue and equipment are the necessary material basis for students to learn physical education courses, and are the material conditions for completing the corresponding teaching goals and integrating into cultivate morality physical education thoughts. In the early days, the Chinese women's volleyball team used all available equipment in a 28-day bamboo shed at the training base in Zhangzhou, Fujian[3]. Athletes and coaches make full use of existing sports venues and equipment to train for themselves, for volleyball, and for the motherland. Among the physical education courses, most subject-based physical education courses are conducted in schools, and the venues and equipment are guaranteed. However, in some regions and schools, the development is uneven and the corresponding equipment is lacking. In the subject-based physical education curriculum, teachers and students select existing equipment together to meet the existing teaching environment and conditions, and cultivate students' motor skills and sports skills and interest. In active physical education courses, the participants are diverse, the number is large, and the space is broader, which has more requirements for sports equipment and venues. The "teaching" of active physical education courses is not as obvious as that of subject physical education courses. Teachers and students are in a freer condition to carry out activities. Teachers and students can modify equipment or even create new sports equipment and rules according to their needs. Under such free conditions, without the rigid requirements of physical education, teachers and students can better integrate with each other, stimulate students' creativity, and create a harmonious teacher-student relationship. Lang Ping once said: The girls and I are more about getting along with you as friends than just a relationship between coaches and athletes. It is also with this kind of friendship similar to friends, a harmonious team atmosphere can become the fertile ground for creating the spirit of the women's volleyball team. A good teacher-student relationship and a harmonious classroom atmosphere can make teaching more effective with half the effort, and provide a guarantee for completing the teaching goals and realizing the value of the physical education curriculum.

\subsubsection{Diligently seek to improve subject knowledge}

The active and enterprising spirit of the women's volleyball team stimulates teachers and students to learn academic knowledge together. Premier Zhou Enlai kindly invited Dasong Bowen to come to China for guidance. In the following two years, Dasong Bowen came to China three times to bring the concept of "strict requirements, obey orders, and cultivate team spirit" to China, integrate into the women's volleyball team, and make it through solid training. It is deeply integrated into the consciousness level of women's volleyball players, establishing and gradually cultivating the earliest "women's volleyball spirit"[3]. Put aside prejudices, learn from excellent fields, and continue to be aggressive. This is one of the inspirations from Dasong Blog's coming to China, and it is also the spiritual wealth given to us by the spirit of the women's volleyball team. Subject knowledge is only a tool to accomplish the goal of "Lide Shuren" in the physical education curriculum, and make full use of this tool to complete the physical education curriculum goal. Nowadays, more and more attention are paid to physical education, and society is more and more able to understand the importance of sports. However, the traditional ideas of "developed limbs and simple mind" and "what's the use" of sports are still one after another. Teachers must establish their own image in physical education courses, not only in having complete professional knowledge and sports skills, but also in breaking disciplinary barriers, learning other scientific and cultural knowledge, and improving their own cultural literacy. The development of information technology drives the change of teaching technology. The application of modern multimedia can make the physical education curriculum more complete. Some introductory teaching content courses can be taught through multimedia teaching aids, which can enable students to understand sports more intuitively and enhance students' perception of sports culture knowledge. Whether in the subject physical education curriculum or in the active physical education curriculum, teachers should teach students relevant physical subject knowledge and safety knowledge, and guide students to explore sports-related knowledge and safety protection knowledge. On the one hand, through the teacher's answers, the image of a physical education teacher is further established. On the other hand, it can enable students to actively learn physical education knowledge, cultivate students' perfect knowledge system of physical education, and give full play to the value of physical education curriculum.

\subsection{Target of Human and Self Development}

Physical education curriculum is the content carrier for students to learn sports skills and improve their physical fitness. It is the core content of physical education activities and the fundamental source of cultivating students' core literacy[4]. Incorporating the value orientation of women's volleyball spirit into the study of sports skills in the physical education curriculum can better cultivate students' sports health literacy, sports attitude, and sportsmanship. The physical education curriculum has a huge and rich content system with a high degree of integration and flexibility. Under the influence of implicit requirements such as rules and order in the physical education curriculum, students are nurtured and developed in moral behaviors of restraint, self-discipline, unity and harmony[5].

\subsubsection{Physical education courses face up to physical skills}

In the physical education curriculum, the learning of sports skills is essential, which is also one of the goals of physical education. Many scholars now scoff at the purely teaching of motor skills in physical education courses. However, it is undeniable that as a component of school sports, physical education curriculum has an indelible contribution to the 
process of cultivating students' sports skills, physical fitness, and developing lifelong physical education awareness. The core task of physical education class is to teach students sports skills, and at the same time guide students to use the learned sports skills to exercise their bodies and cultivate the core qualities of sports disciplines[6]. The basic technique is the foundation of the sport. The basic technique of passing, padding, buckling, serving and blocking are essential factors for volleyball. The women's volleyball team was able to return to the championship stage after five consecutive years of brilliance and silence, which is an emphasis on basic skills and athletic ability. While cultivating students' motor skills in the teaching process of subject-based physical education courses, using the spirit of the women's volleyball team as a guide to correct skills learning attitudes and structured sports skills teaching can better enable students to master motor skills. Dong Cuixiang concluded in the article "Theoretical Foundation and Practical Basis for the Establishment of the Key Points of China's Healthy Physical Education Curriculum Model" that the structured teaching of sports skills should have the characteristics of integrity, relevance, hierarchy, and application. It can be seen that the structured teaching of motor skills does not allow students to learn a single technique, but the practice and application of sports knowledge, motor skills, physical fitness, rules, multiple techniques and combined techniques. Active sports courses can be targeted and appropriately arranged for some professional skills. These sports skills are the advancement of the teaching skills of disciplinary sports courses. Students who have the ability to learn and are interested and gifted in the sport can further study. In this way, the subject-based physical education curriculum and the active physical education curriculum complement each other, so that the unity and personality of the students can be taken into account, it can also make the function of P.E. curriculum to better infiltrate the students' basic technical learning process.

2.3.2 The spirit of women's volleyball practice sports curriculum

The spirit of the women's volleyball team conveys the power of hard work, which is manifested in three dimensions, one of which is "static" self-struggling. It is very monotonous and even painful to learn the basic skills of volleyball to pass, pad, buckle, serve and block. Monotony refers to the practice of repeating a single action. Taking the ball as an example, fixing the hand shape, raising the wrist and straight arm, and controlling the strength can all be sublimated in repeated exercises over many years. The pain is not only manifested physically but also psychologically. Only when a certain load is reached can the body be stimulated, and psychological pain will continue to increase in the process. This kind of struggle is one's own struggle, whether it is a 100-time or 300-time breakthrough. Whether it is a subject-based physical education curriculum or an active physical education curriculum, while taking into account the individuality of the students, it is also necessary for the students to make certain breakthroughs. Teachers design teaching plans before class and train them with certain quantitative standards. This kind of self-struggle is observable for teachers and controllable for students themselves.

The second is the "individual" self-struggle. In a volleyball game, individual save actions such as roll saves, dive saves, etc. It demonstrates the active and enterprising fighting spirit, and is the individual's responsibility to the sports team. In the physical education curriculum, this kind of individual self-struggling requires active guidance from teachers, and the use of appropriate teaching methods and teaching skills to stimulate students' fighting spirit. This kind of "individual" struggle is more difficult for teachers to control and organize students individually. The students themselves are susceptible to self-emotions and have certain safety hazards.

The third is "collective" struggle. In the face of a powerful opponent, it is not to shrink back, but to face the bravery and fearlessness. At the 2014 World Championships in Italy, the Chinese women's volleyball team entered the final without support. In a duel with the US team, the Chinese women's volleyball team lost 25:27 and 20:25 in the first two games, forming a 0:2 situation. In the third game, the women's volleyball players competed for every ball, and finally pulled back a game with an absolute advantage of 25:16, forming a 1:2 situation. Although the team still lost to the United States in the fourth game and lost the championship, the spirit of collective struggle, reluctance to admit defeat, and fear of losing demonstrated by the women's volleyball players is still worth learning. In the subject-based physical education curriculum, this kind of collective struggle requires teachers to create an appropriate teaching atmosphere, teach in groups, and stimulate students' fighting spirit based on the sense of group honor; In active physical education courses, competition and game teaching methods can be used more often.

"Static" self-struggle is the individual's struggle for technology, "Individual" self-struggle is more from the struggle for the collective sense of belonging and honor, The "collective" struggle is the sublimation based on the static self-struggle and the individual's self-struggle, and it is a breath of energy gathered by each individual. These three struggling dimensions are applied differently in the teaching process of physical education. Teachers should choose different dimensions reasonably in order to better play the guiding role of the spirit of women's volleyball in the physical education curriculum.

\subsubsection{Women's volleyball team spirit cohesion and unity classroom}

Volleyball as a team project is inseparable from the collective consciousness and solidarity of this project. The women's volleyball team, as the representative of China and even the world volleyball, must have a unique innate spirit. The unity and cooperation shown by the spirit of the women's volleyball team is not only a grand sentiment, but also embodied in specific details. When preparing for the event, the women's volleyball team shouted the slogan "Come on" together; Serving and shouting "good shot" during the game; Encourage each other when scoring offense. These subtle details can reflect the spirit of solidarity and cooperation of the women's volleyball team. These are of reference significance in physical education courses. For example, in subject-based physical education courses, students chant slogans together in warm-up activities; When doing skill movements, imitate and observe each other, encourage them 
in time, etc. In the active physical education curriculum, strengthening the training of the awareness of the rules, on the one hand, can enhance the student's team consciousness and collective spirit; On the other hand, affected by the nature of active physical education courses, student organization and discipline work are cumbersome, and strengthening the awareness of rules can better organize. These teachings are not only a means, but also a rational and self-conscious running through the physical education curriculum. The consciousness of curriculum rationality should adhere to the unity of subjective and objective standards, the unity of subjective and objective rationality, the unity of instrumental rationality and value rationality, and the unity of subject demand and value generation[7]. In the physical education curriculum, this kind of rational consciousness is derived from the attributes of physical education curriculum itself. Physical education curriculum is an integral part of education, and its fundamental attribute is to "educate people." Adhere to the student-oriented thinking in teaching activities, The establishment of teaching goals should be based on the teaching object and the teaching environment meets the needs of the subject; Gradually establish harmonious interpersonal relationships among students, improve students' social communication ability and social adaptability, and cultivate students' good sports style and cooperative spirit.

Since the 20th century, our country's school physical education curriculum reform has developed to a new stage, and new explorations have been carried out. On the basis of the value orientation of "health first", more and more emphasis is placed on students as the main body, emphasizing students' innovative spirit and practical ability, It not only pays attention to the teaching of professional knowledge of sports disciplines, but also pays attention to the overall development of students, It embodies the value orientation of "student-oriented"[8]. Under the guidance of this value orientation, In the physical education curriculum, the spirit of women's volleyball is the main line. In the process of physical skills learning, students' physical health literacy will be enhanced, and students will be cultivated to strive for progress, unity and cooperation, and sports attitudes to highlight students' dominant status, which is more in line with the requirements of physical education courses in the new era.

\subsection{People and Society Advocate Socialist Core Values}

Judging from the original intention of "Lide Shuren". The purpose of "Lide" is to root "morality" in the heart, words and deeds of the educated through moral practice, make it grow into a moral and virtuous socialist builder and successor through receiving education[9]. The physical education curriculum is undoubtedly a practical foothold in school education. The physical education curriculum is a microcosm of society, Through the learning of sports skills and the cultivation of sports ethics, students will gradually improve their personalities and enable them to have competitive, cooperative, and good communication skills. The educational values of sports refer to people's understanding of the positive role of sports in transforming people or social groups' ideologies and social behaviors. It is about the value judgments and basic viewpoints of sports in cultivating and transforming people[10]. With the core socialist values as the core and the spirit of the women's volleyball team, the core sports literacy can give full play to the role of the physical education curriculum. In the spirit of women's volleyball team, the tenacious fighting spirit, the harmonious spirit of unity and cooperation, the selfless dedication without seeking return, the diligent patriotism and professionalism. It not only enriches the connotation of sports core literacy, but also enables students to better experience these spirits in physical education courses, establish a good moral character for students, so that students can better practice the core values of socialism from the inside out. Do a good job in sports cultural exchanges and sports cultural inheritance in subject-based sports courses. Use existing public sports services in active sports courses, watch sports games in sports parks, gymnasiums, or provide volunteer services in school sports meets. The integration of the spirit of the women's volleyball team not only positively changes the mental outlook of the students, but also improves the entire humanistic environment and even the institutional environment of the school. Then the school's good humanistic and institutional environment is further edifying the students, forming a virtuous circle of women's volleyball spirit shaping students-students creating a positive campus culture-campus culture nurturing students.

\section{Conclusion}

Many people with lofty ideals have devoted themselves to the cultivation of core literacy, different disciplines have their own unique core literacy. The establishment of the sports core literacy model provides a basis for the cultivation of core literacy in the physical education curriculum, the integration of the spirit of the women's volleyball team will make the core literacy of sports more specific, and teachers will also have clearer ideas in the process of first-line teaching. The superiority of spiritual culture lies in the inheritance of human cultural genes and the ability to be continuously enriched and perfected in practice. The spirit of the women's volleyball team has formed a precious spiritual culture through inheritance and improvement. The inheritance of this spiritual culture is not only reflected in the inheritance of the strength of the "old women's volleyball team" to today's younger generation of players, but also in the practice of physical education.

\section{References}

[1] Yang Zongyou, Xia Siyong. Study on the cultivation of sports core literacy in the multiple curriculum implementation mode $[\mathrm{J}]$. Journal of Guangzhou Sport University, 2019, 39(05): 111-115.

[2] Zhao Feng Xia, Cheng Chuan Yin, Zhang Xinhui, Li Juhong. Research on the construction of sports core literacy model[J]. Sports Culture Guide, 2017(01): 154-159.

[3] Liu Guangyi. The value and significance of integrating women's volleyball spirit into college volleyball teaching[D]. Hebei Normal University, 2016.

[4] Qiquan Zhong. Curriculum development based on core literacy: challenges and issues[J]. Global Education Outlook, 2016, 45(01): 3-25.

[5] Zhao Fuxue, Huang Guisheng, Li Cheng, Shi Ying, Du Hongwei. Theoretical interpretation and practical appeal of the ideological and political construction of physical 
education courses from the perspective of "Lide Shu ren"'J]. Physical Education Research, 2020, 34(5):7.

[6] Dong Cuixiang, Lu Huimin. Theoretical basis and practical basis for establishing the key points of China's health physical education curriculum model[J]. Sports Science, 2020, 40(06): 24-31.

[7] Zhang Huanyu, Zhang Qiang. Combing and enlightenment of the value orientation of school physical education curriculum in China[J]. Sports Culture Guide, 2015(11): 131-135+139.

[8] Wang Deru. Research on the value orientation of curriculum cultural consciousness[J]. Educational Research, 2006(12): 72-78.

[9] Zhang Xu. Benevolence education: Exploration of a new paradigm of morality and cultivation in the new era[J]. Chinese Journal of Education, 2019(10): 96-97, 103.

[10] Chen Qi, Yang Wenxuan, Liu Haiyuan, Lu Changfen, Deng Xinghua, Zhang Xiqian, Feng Xia. Research on contemporary sports values in my country[J]. Sports Science, 2006(08): 3-9+74. 